\title{
Dietary management of liver disease
}

\author{
By E. Carol Bateman, Royal Free Hospital, Pond Street, London, $N W_{3}$
}

This paper is only intended to present a brief outline of the benefits, or otherwise, of using a modified diet to treat the symptoms of those liver conditions most commonly met with in the clinical situation. Liver disease may have a nutritional origin, as in the fatty liver seen in kwashiorkor, when the diet has contained sufficient energy in the form of carbohydrate but is deficient in protein. The fatty liver of obesity or of mismanaged long-term parenteral nutrition, is also related to malnutrition. The provision of a diet containing sufficient amino acids may reverse these conditions if the diet can be instituted before the liver damage is too severe.

Alcoholic cirrhosis was long thought to be particularly prevalent in those alcoholics who took an inadequate diet, but it now seems more likely that alcohol is itself a toxic agent and those who are susceptible will sustain liver damage regardless of whether their diet has, or has not, been nutritionally adequate.

Women, for example, appear to be more at risk from the toxic effect of alcohol than men are (Morgan \& Sherlock, 1977).

It has been recognized for centuries that the liver is fundamentally concerned with the digestion and metabolism of food. Diseases of the liver are often accompanied by anorexia, nausea, vomiting and food intolerance and it is therefore not surprising that a variety of dietary measures have been advocated in their management, and indeed are still advocated today. The dietitian discussing dietary modifications with patients will hear many patients say they have been advised to avoid spicy foods because of their liver disease, and many patients, especially from the Middle East, will insist that their doctor has told them they must not eat citrus fruit, presumably on the grounds that the yellow colour of the fruit may increase the colour of their jaundice. There would appear to be little scientific evidence behind either of these dietary restrictions.

For most patients with liver disease, other than those liver diseases of nutritional origin, dietary treatment is aimed at relieving the symptoms of the disease and there is little reason to suppose that modifying the diet will cure the underlying disease. It is important to remember this before imposing a diet which may be unpleasant for the patient and difficult to comply with. The aim of the diet should be to improve the quality of life for the patient, and any modified diet advised should be as pleasant and tolerable as possible. The three most usual symptoms, or complications, of liver disease for which we may wish to instigate dietary measures are jaundice, ascites and hepatic encephalopathy. 


\section{faundice}

Jaundice is a common finding in liver disease and may be present for many different reasons. A low-fat diet is frequently ordered for the jaundiced patient, regardless of the cause of the jaundice, but there is no reason to restrict dietary fat intake unless the patient does not tolerate fat well. If jaundice occurs as a result of a familial condition such as Gilbert's Syndrome, or familial unconjugated hyperbilirubinaemia, fat intake should not be restricted. A diet low in fat and high in carbohydrate may cause an elevation of unconjugated bilirubin in the Gilbert's Syndrome patient, whereas an iso-energetic diet high in fat may cause a reduction in the unconjugated bilirubin and a consequent decrease in jaundice (Gollan $e t$ al. 1976).

The jaundiced patient with infective hepatitis should be advised to eat as normally as possible. There is no need to restrict fat to below the normal level of about $35 \%$ of the total energy intake. A diet reasonably high in protein is advisable for the patient with hepatitis who is not in liver failure, but there would appear to be no advantage in trying to increase dietary protein to a level higher than $1 \mathrm{~g} / \mathrm{kg}$ body-weight (Sherlock, 1973).

Where jaundice occurs as a result of chronic cholestasis, as in primary biliary cirrhosis, or more properly chronic non-suppurative destructive cholangitis, there is a deficiency of bile salts in the intestine and in consequence fat may be poorly absorbed and badly tolerated. The cholestatic patient passes frequent pale, bulky stools and restricting dietary fat may make life more tolerable for the patient, simply by reducing the quantity and frequency of stools. As bile salts and cholesterol are unable to enter the gut, blood levels rise and cholesterol may be deposited as xanthalasma around the eyes and in xanthomas around the hands, elbows, buttocks, calves and other parts of the body. The distressing pruritis suffered by many of these patients has been thought to be due to the high level of bile salts in the blood, but in the terminal disease, when bile salt levels may be even more greatly increased, the itching frequently disappears. Cholestyramine, which acts as an exchange resin with bile salts, may be very helpful in controlling itching. However, cholestyramine also increases steatorrhea and should therefore be given in the smallest possible amount needed to control the itching. Cholestyramine has the added disadvantage of being unpleasant to take, often causing nausea and depression of appetite. This may be a problem when trying to persuade these often distressingly thin patients to eat more.

Fat soluble vitamins are not well absorbed by patients with cholestasis and must be given by injection on a regular basis, usually monthly injections. Osteomalacia, resulting in bone pains and fractures is a common feature of biliary cirrhosis, usually when the disease has reached a late stage. Monthly prophylactic intramuscular cholecalciferol and calcium supplements may help to prevent this. Some benefit has been claimed from the use of oral 25-hydroxy-vitamin D (Wagonfeld et al. 1976) and some histological improvement of bone lesions has been seen with intramuscular 1,25-dihydroxycholecalciferol (Long, 1977). 
In practical terms, therefore, the cholestatic patient may benefit from a restriction of dietary fat to about $30-40 \mathrm{~g} / \mathrm{d}$. This restriction will help to reduce faecal fat and may help $\mathrm{Ca}$ absorption, as fat in the gut of cholestatic patients may form insoluble soaps with calcium. Many of these patients are extremely thin and medium chain triglycerides (MCT) may be added to the diet to increase the patient's energy intake. The advantage of MCT is that they are water soluble and may be absorbed directly into the blood-stream without the presence of bile salts in the gut. A milk powder in which the milk fat has been replaced by MCT is particularly useful in these diets as a source of calcium. The MCT-filled milk powders available in the UK have the added advantage that they have a low sodium content and can be given to patients with ascites, a frequent additional complication in primary biliary cirrhosis.

\section{Ascites}

A second feature of liver disease for which dietary measures may be instigated is ascites, that is the retention of large quantities of fluid in the abdomen.

The most important factors in the development of ascites are the failure of the liver to synthesize albumen and hence a lowered plasma osmotic pressure, and portal hypertension. More fluid enters the peritoneal cavity than leaves it and hence ascites develops. This results in depletion of effective body fluids, which causes the renal tubules to retain $\mathrm{Na}$ and water. The distal effect is presumably due to aldosterone action, the mechanism of the proximal action is uncertain.

The use of one or more diuretics will probably be necessary, but diuretics must be approached with caution, as over-enthusiastic early use of diuretics can lead to renal failure (the hepatorenal syndrome). The kidney retains $\mathrm{Na}$ avidly and a diet low in $\mathrm{Na}$ is very important; the renal tubules may reabsorb as much as $99.5 \%$ of the filtered load of Na.

Initial treatment of the ascitic patient is therefore bed-rest and a low-Na diet. This regime alone may be sufficient to cause a diuresis. The diet should be as low in $\mathrm{Na}$ as possible, but if protein intake is to be kept up to $60-70 \mathrm{~g}$ it is difficult to get the $\mathrm{Na}$ content of the diet below $20 \mathrm{mmol}$, without using a low-Na, highprotein supplement which the patient may be unwilling to accept.

Many ascitic patients may have to remain on a low-Na diet for a considerable time and it must therefore be possible for them to manage the diet at home, and it must be as palatable as possible. All food is cooked without salt and no salt is added at table. Salt substitutes may be used, provided there is no renal problem with potassium retention, but in practice, salt substitutes prove a disappointment to the majority of patients. The patient has to learn from the start that there is no real substitute for salt and that he must adjust his outlook in order to enjoy his food in a different way. The hardest thing for most people to accept is salt-free bread and the dietitian may have to compromise by allowing a small amount of ordinary bread in the diet. As the average slice of commercial bread in the United Kingdom contains more than $5 \mathrm{mmol} \mathrm{Na}$ this compromise may increase the $\mathrm{Na}$ content of the diet considerably. 
It is important that the ascitic patient understands that the weight loss on which he may be congratulated while an in-patient in hospital is 'water' weight. Some patients will avoid bread, potatoes, fried foods etc. on the hospital menu in the belief that weight loss is desirable, nobody having taken the trouble to explain to them the difference between the weight loss caused by a diuresis, and weight loss due to a low-energy diet.

For many people with persistent ascites diuretics combined with a low-Na diet is at present the most efficient way of controlling the amount of fluid in the abdomen. For some, intermittent use of the so-called Paris pump may help considerably, but a low-Na diet will usually be required as well. The Paris pump works on a system of ultrafiltration, removing excess fluid and electrolytes, but returning the protein fraction to the body. There is thus some long-term effect, as the plasma osmotic pressure is raised by the return of the concentrate to the circulation. (Parbhoo et al. 1973.)

The LeVeen shunt (LeVeen et al. 1974; Lancet, 1978) is another possible method by which ascites may be controlled. A thin tube, with a valve which rests in the peritoneal cavity, is wriggled up under the skin to the neck, to open into the jugular vein. The pressure built up by ascitic fluid in the abdomen causes the valve to open and allow the fluid to run up the tube and into the neck vein, thus restoring plasma volume and preventing the renal tubules from retaining large amounts of $\mathrm{Na}$. Unfortunately, the shunt does tend to get blocked, which limits its usefulness at present with some patients.

A low-Na diet is not very easy to comply with, but with some imagination it can be made reasonably palatable, and the majority of patients will accept the $\mathrm{Na}$ restriction as being preferable to the discomfort of severe ascites.

\section{Encephalopathy}

The third aspect of liver disease for which a dietary management may be necessary is hepatic coma and chronic portal systemic encephalopathy.

The mechanism of portal encephalopathy is not clear, but very simply it would appear that the damaged liver cannot clear the toxic substances produced by the action of intestinal bacteria on ingested protein. What is not known is precisely which the relevant toxins are. It is certainly true that a low-protein diet will cause a rapid improvement in the mental condition of the encephalopathic patient.

If a patient is actually in hepatic coma, perhaps as a result of hepatitis, or of some chronic liver disease, all dietary protein is stopped, and only glucose fluids given. As consciousness increases, protein is added to the diet in 10 to $20 \mathrm{~g}$ increments at intervals of a few days, until the upper limit of protein tolerance is reached, or until the patient is on a normal diet. Protein in the diet should be kept as high as possible, while avoiding the signs of encephalopathy. These signs may include an increase in foetor, in liver flap or confusion, or may be detectable on electro-encephalography, as the number of cycles on the EEG diminish with increasing coma, from the normal of approximately 9 cycles/s. The use of psychometric tests, such as the Reitan Trail Test, may also be a good guide to the 
level of consciousness. For this test the patient has to join numbers, arranged randomly, together in the correct order within a given time. The time taken to perform this test varies according to the degree of encephalopathy, so that repeating the test at intervals may give a good guide to the patient's current state. Patients with chronic portal systemic encephalopathy may be able to tolerate less than $40 \mathrm{~g}$ protein/d, although toleration of dietary protein can be enhanced by the administration of neomycin, a non-absorbable antibiotic, and of the synthetic disaccharide lactulose. (Elkington et al. 1969; Bircher et al. 197r.)

The pathogenesis of chronic portal systemic encephalopathy is not yet clear, but there is considerable evidence that methionine and its intestinal breakdown products, the mercaptans, are important factors. Large doses of methionine given by mouth may induce encephalopathy in marginally compensated cirrhotics, whereas comparable doses of methionine given intravenously have no such effect. Mercaptans have been identified in the breath of cirrhotic patients and the concentration seems to correlate with the degree of foetor hepaticus. (Chen et al. 1970.)

There is a change in the proportions of plasma amino acids in patients with liver disease, with higher than normal values for the aromatic amino acids phenylalanine and tyrosine, and to some extent tryptophan, and lower than normal values for the branched chain amino acids, leucine, isoleucine and valine. However, it would appear that the change in proportions is due to liver damage, and is not a cause of encephalopathy. (Morgan et al. 1978.)

It has been suggested that the poorly compensated cirrhotic may tolerate more protein if it is from vegetable, not animal, sources (Greenburger et al. 1977). The vegetable-protein diet is lower in methionine than the animal-protein diet, and the lower levels of aromatic amino acids might possibly also be beneficial. A vegetableprotein diet was therefore tried for a few patients at this hospital, but there seemed to be little benefit to most patients and the diet was not well accepted.

The vegetable-protein diet tended to be very bulky and when the protein level was as high as $60-70 \mathrm{~g} / \mathrm{d}$ it was very hard to persuade patients to eat the full amount. An additional complication was that the diet for most of the patients also had to be low in $\mathrm{Na}$ which made it even less palatable.

At present, it would seem that the best management we have for chronic portal systemic encephalopathy is to give a low-protein diet, of mixed proteins of the type the patient is accustomed to eating, with the addition of neomycin and lactulose. Many of these patients are ill and confused, and may have social problems. The prescribed diet must therefore be as easy to comply with and as manageable as possible. As every dietitian is well aware, no diet works unless the patient eats it.

\section{REFERENCES}

Bircher, J., Haemmerli, U. P. \& Scolow-Lavizzari, G. (1971). Am. F. Med. 51, 148. Chen, S., Zieve, L. \& Mahadevan, V. (1970). f. Lab. clin. Med. 75, 628.

Elkington, S. G., Flock, M. H. \& Conn, H. O. (1969). New Engl. f. Med. 281, 408.

Gollan, J. L., Bateman, C. \& Billing, B. H. (1976). Gut 17, 335 . 
Greenburger, N. J., Carley, J., Schenker, S., Bettinger, I., Stamnes, C. \& Beyer, P. (1977). Dig. Dis. 22, (10) 845 .

Lancet, Editorial (1978). i, $3 \mathrm{II}$.

LeVeen, H. H., Christoudias, G., Ip, M., Luft, R., Falk, G. \& Grossberg, S. (1974). Ann. Surg. $180,5^{80}$.

Long, R. (1977). 7. hum. Nutr. 31, (2), 115.

Morgan, M. Y. \& Sherlock, S. (1977). Br. Med. f. I, 939.

Morgan, M. Y., Milsom, J. P. \& Sherlock, S. (1978). Gut 19, (I 1), 1068.

Parbhoo, S. P., Ajdukiewicz, A. \& Sherlock, S. (1973). Gut I4, 421 .

Sherlock, S. (1973). In Diseases of Liver and Biliary System, 5 th edn. Oxford: Blackwell.

Wagonfeld, J. B., Nemchausky, B. A., Bolt, M., Horst, J. V., Boyer, J. L. \& Rosenberg, I. H. (1976). Lancet ii, 391 . 